\title{
Information Sharing with Data Exchange Package
}

\author{
Zhen Wang $^{1, \text { a }}$, Hong-Xia Zou ${ }^{2}$ and Bu-Qing Zheng ${ }^{1, b}$ \\ ${ }^{1}$ Department of Graduate, Equipment Academy, BeiJing 101416, China; \\ ${ }^{2}$ Department of Information Equipment, Equipment Academy, BeiJing 101416, China \\ awangzhen921206@126.com ${ }^{\mathrm{b}} \mathrm{m} 15201672978 @ 163 . c o m$
}

Keywords: data exchange package, information sharing, TDP, COI, enterprise information management.

\begin{abstract}
Boost expansion of enterprise cooperation require a new method to exchange data among systems and shield systems difference and shield data format difference. TDP (Technical Data Package) is designed for enterprise to manage data with product lifetime, and provide a method to integrate and manage data with product model. NIEM (National Information Exchange Model) use data component to model object and concept in real world to provide a common understanding of information. Using NIEM to model things and integrate data with TDP could provide a new method to shield systems and data format. This paper design a data exchange package format and make rules to extend the function of TDP to manage all data for enterprise cooperation.
\end{abstract}

\section{Introduction}

Enterprises has adopt many systems for its informatization and deploy it into every corner of its manufacturing factory, designing laboratory and management department. For most of enterprises those systems are development by different software company, which result in a problem to combine all those information for developers, managers and those customers, who asks the production information for the product they brought. Nowadays an large equipment production need the cooperation between enterprises and designing laboratory, which ask product`s information rapidly transmit from one terminal to another for cooperation design, real-time monitor, technical problem feedback.

The traditional way of information sharing between enterprises is to install an agent between two systems, this method is easy to deploy and high efficiency when developed in several systems especially no more than four systems. Another way is to come up with a new standard for information sharing, this method ask all those enterprise to apply the same standards, it will be easy for information sharing because all systems use the same format of data, but it will not be easy for support all the request of information sharing. Because of such a shortcoming of traditional methods, we try to find a way for information sharing of easy to deploy and be able in massive systems.

\section{Theory of Information Sharing}

\subsection{Technical Data Package}

TDP is the abbreviation of Technical Data Package. It was defined by MBE as "TDP is a technology for supporting procurement, production, engineering technical explain, logistics supports (such as configuration of engineering data, training and technical manual), this description defines the required design configuration and/or performance requirements along with the procedures required to ensure adequacy of item performance[1]. The TDP consists of applicable technical data such as models, drawings, associated lists, specifications, standards, performance requirements, quality assurance provisions, software documentation, and packaging details [2].

The most benefit of TDP is the new method for data integration. The traditional way of enterprise technical data management is to sort 2D graphics and documents into a manual by product or important part of product. The manual will be transfer into upper or lower enterprise, and help those 
enterprise to search and get copy of information. For multi-enterprise-cooperation, even if this method is tolerable for its efficiency, it is not acceptable for its data incompleteness even if different version of information. TDP provide a method to integrate data with product model, which could integrate different department to maintain the uniformity of data

\subsection{Community of Interest}

Community of Interest (COI) is a group of organization or people who have the same interest, and was came up in the year of 2003[3]. COI has been taken into information sharing by the organization of NIEM (National Information Exchange Model) to divide a whole information sharing environment into several part, for each part take the same standard to communicate, the communication between parts using an agent to transmit the format from the source part into the target part.

Using the theory of COI, the whole environment of information sharing can be divided into several part. In the environment of manufacturing, we use the manufacturing mission to divide it. For each manufacturing mission, there is a master enterprise and several second-level enterprise and even some third or more level enterprise. The master enterprise`s duty is to manage the whole COI group, when the master enterprise ask for a part of this manufacturing mission by supply chain to the second-level enterprise, the master enterprise stipulate which information and the format of those information should be submit. Those information of product is from product s beginning lifetime, from designing, simulation, test, production, quality testing, to the delivery.

\subsection{Community of Interest Data Modeling}

Data Modeling could describe the relationship of data in information sharing by models to achieve the same understanding of information. The NIEM use data components to describe the relationship of exchanging data [4]. The data components are source of real world`s different object and concept, which made it is easier to understand.

One essential problem for data component to deal with is the different domain use the same identify of object but the property is different. To solve the problem, the NIEM came up with a method of Core Model and Domain Model. The Core Model contain the universal object and its universal property, and every domain every terminal of information sharing environment must support the Core Model. The Domain Model describe different domain and its object [5].

\section{Design of Information Sharing}

The advantage of TDP, based on product model to integrate data, is an optimal method for multi-systems, multi-domains information sharing based on realistic object. The TDP could be fixed to be the carriage of enterprise information sharing, to support information sharing in product design, simulation and test, technology design, product production, combination and quality test, all steps in product lifetime management, to meet the need of product lifetime management, enterprise business management and production management, to support information sharing between departments and enterprises among supply chain.

\subsection{Structure Design}

Principle of design data exchange package is standardize data packaging, structuralization components and original format data, and the structure of data exchange package are showed in Fig. 1.

Standardize data packaging request a simple but unification structure. To meet this need, the structure of data exchange package should be as simple as it can, so the structure just keep the necessary information for exchange and routing. 


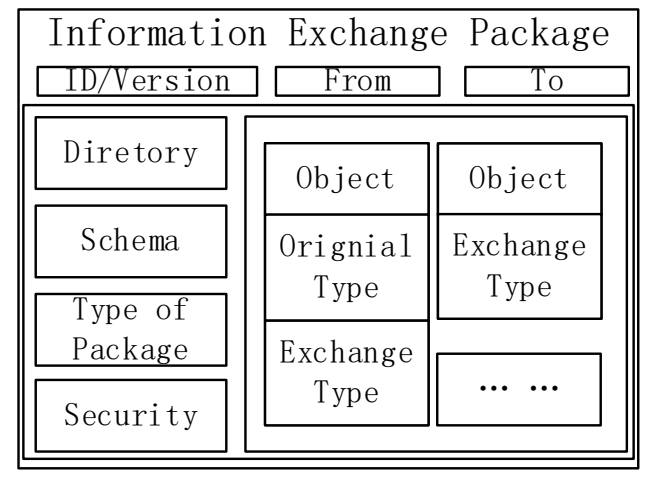

Fig. 1 Structure of Data Exchange Package

Structuralization components could help receiver to quickly understand the package and find the data needed. For this purpose, a directory is needed first, the directory just give information of what information does the data exchange package contain and for each information what the data format and version is, to achieve the function that receiver not analysis the data exchange package fully but can got what the package contain. Schema file is the key part of whole data exchange package, the schema file simplify the structure of those information, the relationship and connection between each information, with the schema file, receiver could understand relation of information, when the information is update or delete, what operation should be done to other information to maintain the completeness of all the information in data exchange package. The type word is designed for distinguish different information sharing mission and clarify the purpose the package is designed for. Safety word is to keep the package with its safety, authority and completeness, the data of information could be encipherment together or single just record it in safety word, the message digest is also included in safety word.

Original format data is the special of data exchange package. For all information sharing, data format transformation is a necessary step, but data distortion and data missing is more likely to happen especially in multi-systems information sharing. The original format data is been extract from source system, without any data dealing, seal into the data exchange package (or just record the link of original format data, when it is enormous not fit the data exchange package). Usually, receiver extract those data that is suitable for its system or the middle format for exchange, but when receiver find something is wrong with the data or it is unreliable, it can extract the original format data to be the authoritative data to get the information.

\subsection{Rule of Information Sharing Environment}

Principle of design data exchange package is one package meet all needs, which means the data exchange package should pack all the information and its necessary format of data into one package and publish it. For receiver, to extract the information and the suitable format data for necessary. This method make it easier to manage the information sharing environment and the information resource.

To use data exchange package for information sharing, few steps should be simplified. 1, information sharing environment establishing, to establish the information sharing environment, all systems in the environment should accept the method of data modeling with data component, all systems should accept the Core Model. 2, group systems with information sharing mission, when a new need of information sharing appear with a product, the master enterprise group lower level enterprise to a COI, when the COI set up, a list of information provided and required by systems should be listed, to confirm all systems sending and receiving information and its data format. 3, information sharing management, the master enterprise manage the whole information sharing environment, and maintain the environment running normally.

\subsection{Rule of Data Exchange Package}

Schema and Authorization: The data exchange package contain many information and data, but one systems may not allowed to extract all information from the package. For this reason, the receiver of data exchange package receive an authorized schema file, this schema file contain information 
position, what`s more, a key for match the safety word and decode the package, message digest to verify the extracted information and its data format is included.

Data integration and distribution: Because the information are integrated by product models, from the start, the product was divided into few part. When the upper enterprise divide the product model into few parts, it modelling the product and record the relation between each parts, define the property of the whole model, seen in Fig. 2. When a data exchange model was submitted, the upper enterprise extract the product model and model information, seal in its data exchange package, using the definition of whole model property to calculate or extract from lower model information to fill the property of whole model. When data was distribution with supply chain into next step of production, the master enterprise of last step seal all information needed and handle to the next master enterprise, then distribute to the lower level enterprise.

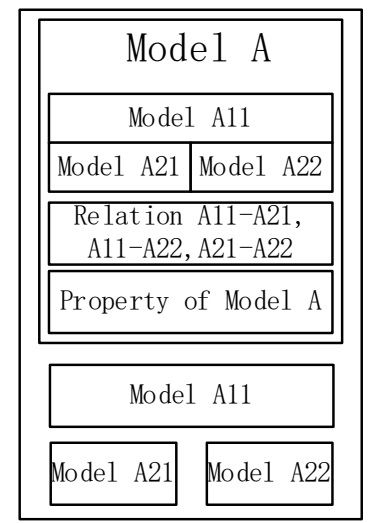

Fig. 2 Model Relation

\section{Conclusions}

Using data exchange package, difference systems and difference format of data could sharing their information together, for each information mission, manager could find an format for all systems or most systems support to be the standard for data exchange, this method reduce the expense of establish an information sharing environment and meet different need, make the environment more flexible. This method could be used in more situation, such as government, and information between different domains.

\section{References}

[1] Information on http://model-based-enterprise.org/model-based-enterprise-overview.html

[2] MIL-STD-31000A, Department of Defense Standard Practice[S]

[3]Gerhard Fischer. Communities of Interest: Learning through the Interaction of Multiple Knowledge Systems.

[4] Information on http://reference.niem.gov/niem/guidance/user-guide/vol1/user-guide-vol1.pdf

[5] Information on https://mise.mda.gov/drupal/node/42 